This item was submitted to Loughborough's Research Repository by the author.

Items in Figshare are protected by copyright, with all rights reserved, unless otherwise indicated.

\title{
Nanotechnology in construction and demolition: What we know, what we don't
}

PLEASE CITE THE PUBLISHED VERSION

https://doi.org/10.1080/20450249.2018.1470405

\section{PUBLISHER}

(C) IOSH. Published by Taylor and Francis

\section{VERSION}

AM (Accepted Manuscript)

\section{PUBLISHER STATEMENT}

This work is made available according to the conditions of the Creative Commons Attribution-NonCommercialNoDerivatives 4.0 International (CC BY-NC-ND 4.0) licence. Full details of this licence are available at: https://creativecommons.org/licenses/by-nc-nd/4.0/

\section{LICENCE}

CC BY-NC-ND 4.0

\section{REPOSITORY RECORD}

Gibb, Alistair, Wendy Jones, Chris I. Goodier, Phillip D. Bust, Mo Song, and Jie Jin. 2019. "Nanotechnology in Construction and Demolition: What We Know, What We Don't”. figshare. https://hdl.handle.net/2134/37098. 


\title{
Nanotechnology in construction and demolition: What we know, what we don't
}

\author{
Alistair Gibb, Wendy Jones, Chris Goodier, Phil Bust, Mo Song \& Jie Jin (2018) Nanotechnology in \\ construction and demolition: What we know, what we don't, Construction Research and Innovation, \\ 9:2, 55-58, DOI: 10.1080/20450249.2018.1470405
}

\begin{abstract}
ABSRACT
Self-cleaning windows, very high strength concrete and thin, super-efficient insulation are just three examples of new building materials promised by nanotechnology, which manipulates matter at the atomic level. But for all their purported benefits, little is known about the risks posed by very small, engineered particles and fibres. Some long and very thin strands might act like asbestos if they are inhaled, for instance. To begin addressing this knowledge gap, the Institution of Occupational Safety and Health (IOSH) sponsored a research team at Loughborough University, led by Professor Alistair Gibb and Dr Wendy Jones, to investigate where these materials are used, how widespread this use is, what the potential risks are and how workers in construction and demolition might manage them. The executive summary of that report, released in January, is reproduced here with IOSH's permission.
\end{abstract}

\section{Introduction}

Nanomaterials are those which have one or more dimensions in the range 1-100 $\mathrm{nm}$. They can occur naturally, can arise as a by-product of human activities such as the use of engines, or can be manufactured. The use of nanomaterials which have been intentionally produced is growing (engineered nanomaterials, ENM, or manufactured nanomaterials, MNM) but it can be difficult to identify exactly where they are and how widely they are used.

The aim of this research is to provide some clarity regarding the current use of nanomaterials in the built environment and the potential benefits and risks arising for those working in the construction and demolition sectors.

Desktop study, including review of academic and manufacturers' literature, has been the main method employed in this research. In addition, interviews have been conducted with individuals ( $\mathrm{n}=$ 59) working in various parts of the construction, demolition and materials supply sectors. Email exchanges and visits to construction, demolition and recycling sites have yielded further data. Data from the different sources have been integrated throughout the report to provide an overview of the current situation with regard to nanomaterials in the built environment.

A second part of this research, published separately and available from Professor Alistair Gibb (a.g.gibb@lboro.ac.uk), has involved laboratory testing of samples of nano-enabled construction products. Some outputs from this have been used in this report to illustrate the nature of the materials involved. Additionally, a summary of the findings from this part of the research can be found in the appendix.

\section{Background}

There are thousands of different nanomaterials, varying in chemical composition, size, shape and many other characteristics. Adding nanomaterials to a product can alter the way that the product performs, sometimes significantly increasing performance or providing additional functionality. 
Nanomaterial use has grown in recent years, and they are used in coatings, electronics, textiles, foods and many other areas. In construction they are used, for example, in concrete to provide additional strength, abrasion resistance, or to reduce the need for steel reinforcement. However, there is little readily accessible information on the extent or scope of their usage. To complicate matters further, the term 'nanomaterial' is used in different ways by different authorities. For example, the ISO definition, which is used in this report, includes all substances which have one or more dimensions at the nanoscale - even if these are internal dimensions. It includes, therefore, nanoscale films, and nanostructured materials which contains no nanoparticles. However, the European Commission (EC) definition is narrower, using the term 'nanomaterial' only in relation to those substances which contain nanoparticles, plates or fibres - collectively known as nano-objects. This definition, intended to underpin any regulation to manage health risks, reflects the concern that increased health risk might arise as a consequence of smaller particle size. However, toxicity varies widely depending on a number of characteristics; for example particular shapes of nano-objects such as long, thin tubes (or rods or wires) are considered to be potentially problematic. Other substances, however, may not be significantly more toxic than non-nanomaterials.

Nano-enabled construction products (i.e. those which have improved functionality as a consequence of the addition of nanomaterials) are rarely precisely labelled. Thus a product which describes itself as 'nano' may contain nanoparticles or other nano-objects; may be highlighting the fact that it is nanostructured (e.g. has nanoscale holes), contains nanoscale films or was developed using nanotechnology; or may be using the term solely in order to make the product seem new and different. Other products may be nano-enabled but choose not to declare this. Consequently, it is difficult to identify those which contain nanomaterials or to determine the exact material involved, and this makes it difficult to assess exposure potential and health risk for those working in construction and demolition.

Nanomaterials within the United Kingdom and Europe are covered by the same regulations as other chemicals and toxic substances, such as COSHH, REACH and CLP, but there are no specific nanorelated requirements except those relating to cosmetics and use in foodstuffs. There are ongoing discussions within the European Commission to refine the definition of nanomaterials and to strengthen requirements under REACH so that better data can be gathered regarding the production and use of nanomaterials which contain nano-objects.

France, Belgium and Denmark have each produced their own regulations requiring the registration of nanomaterials, although the exact requirements vary between them. In the United States, specific reporting requirements for existing and new nanomaterials have recently been approved. Both Canada and Australia manage nanomaterials through their existing registration systems, although the Canadian system makes higher demands, including the classification of a nanomaterial as 'new' even if the bulk (non-nano) form has previously been registered. These regulations generally apply only to nanomaterials which contain nanoparticles or other nano-objects.

\section{The use of nanomaterials in construction}

Research objective: To identify products used in the built environment which contain nanomaterials, and the type of nanomaterials involved

Research findings: The main nano-enabled products available are surface coatings, concrete, window glass, insulation and steel. However, there is a lack of evidence regarding which products definitely contain nanomaterials, and what these materials might be. Current usage rates for nanoenabled products in construction are difficult to assess but appear to be relatively low. 
Concrete: Nanosilicas with various particle sizes can be added to concrete (e.g. silica fume, fumed silica) to produce materials which are stronger, achieve strength earlier or are self-compacting. These have been available for many years but usage in the UK is relatively low.

Titanium dioxide particles provide concrete with self-cleaning properties due to photocatalysis and can also reduce atmospheric pollution. Usage appears to be very limited at present.

CNTs can be added to concrete to improve strength and potentially allow electrical conductivity. CNTs are expensive and difficult to work with: only one CNT-enhanced concrete appears to be commercially available, launched recently following large scale field trials in the United States.

Window glass: Nanoscale film coatings can be added to glass during production to provide special properties for windows. Glass with improved insulation properties (low-e) is quite widely used, especially in the construction of commercial buildings. Coatings can also reduce the amount of solar gain within a building. 'Smart' windows allow solar gain to be varied to accommodate changes in the weather, although these products are currently installed only rarely.

Self-cleaning glass has a nanoscale film of titanium dioxide which breaks down dirt through photocatalysis and is quite widely used, for example in conservatory roofs. Fire safety glass with an intumescent layer of nanosilica has been available for many years but is only used in high-risk situations. When exposed to high temperatures, the nanosilica swells and forms a resilient barrier, absorbing the energy of the fire.

Surface coatings are the most numerous and readily available nano-enabled products for use in the built environment, being marketed for both professional and DIY use as well as being applied to products during manufacture. Many coatings contain silica (either as particles or in polymers) and are marketed for waterproofing or for their easy clean/ dirt repellent properties. Coatings may also contain titanium dioxide nanoparticles, providing selfcleaning properties through photocatalysis; or antimicrobial silver nanoparticles.

CNTs and graphene are novel carbon nano-objects which offer strength, corrosion resistance and conductivity for coatings but only a very small number of such products are commercially available. Carbon black is a much more commonly used (and familiar) material used, for example, for shielding against radio waves. Insulation materials are generally based on silica aerogels, which can be used in insulation blankets, translucent windows or vacuum insulated panels. These materials are highly effective insulators but are expensive and currently not very widely used outside of specialist projects.

Steel can be improved through the use of nanotechnology which modifies the grain structure to improve strength, wear resistance and corrosion resistance. There is some limited use of nanoenabled steels e.g. in reinforcement for road building, although most applications are targeted at the automotive and airline industries.

\section{Health risks from nanomaterials}

Research objective: To summarise the literature regarding the health risks of the nanomaterials most likely to be used in the built environment

Research findings: Potential health risks from nanomaterials are generally associated with the presence of nanoparticles and other nano-objects: the smaller particle size results in increased surface area and therefore increased reactivity. However, the size of objects is not the only factor which influences risk and there are wide variations between different materials in terms of their 
toxicity. This can be influenced by many factors including size, aggregation and agglomeration (how particles stick together), solubility and electrical charge. Particle shape is of key importance: there are particular concerns about fibre- shaped materials such as long, thin and rigid carbon nanotubes (CNTs) and other fibre or wireshaped nano-objects. In some cases, these can behave in the same way as asbestos fibres which are able to travel down to the deepest parts of the lungs but are then too long for the normal clearance mechanisms to remove successfully.

Information currently available about the health risks from nanomaterials is based on laboratory research rather than on cases of ill-health in workers. The results of studies are often inconsistent or inconclusive due to variations in the methods and exact materials used.

Carbon nanotubes are reported to be potentially toxic due to their high aspect ratio and their biopersistency - they are long and thin, and thus similar to asbestos fibres. However, some shapes of CNTs appear to cause no problems; for example CNTs which are short or tangled and nonbiopersistent are less likely to be toxic than those which are long, stiff and biopersistent.

Graphene is another carbon-based nanomaterial; it exists as flat sheets or plates. There is relatively little published literature regarding its health effects. In principle it could be toxic as some forms are able to penetrate deep into the lungs; smaller plates are likely to present a lower risk as they are easier for the body to clear. Carbon black is a particulate nanomaterial which has been used for decades, for example in tyre manufacture. It is classed as a possible carcinogen based on animal studies, although there is no clear epidemiological evidence of this effect. There is some evidence from the literature that carbon black causes irreversible respiratory ill-health, and may be the causative agent behind smoking related ill-health.

Silver is reported to be effective as an antimicrobial in both its nano and non-nano forms. Reports on the health effects of nanosilver particles are inconclusive. There is relatively little evidence to show it is toxic but neither is there sufficient evidence to confirm it as safe.

There is evidence to suggest that nanotitanium dioxide particles may be toxic when inhaled. NIOSH (National Institute for Occupational Safety and Health) in the United States has recommended a maximum workplace exposure level which is one eighth of the exposure level for titanium dioxide which is not nanoscale. There is insufficient evidence to exclude it being carcinogenic. Nanotitanium dioxide is generally considered to be safe for dermal application (e.g. in sun protection creams) although there is limited evidence regarding the impact of preexisting skin damage on this.

Nanosilica is usually amorphous. This is a different form of silica from the crystalline silica which is recognised as a major hazard in the construction industry, particularly as a dust. Amorphous nanosilica particles are acknowledged in most of the literature as being relatively low risk, with any ill effects being largely reversible. However, there is insufficient evidence to declare nanosilica as 'safe', particularly as there are so many different forms of it in use.

\section{Exposure to nanomaterials}

Research objective: To explore the potential for exposure to nanomaterials during construction and demolition

Research findings: The findings presented in this section are based on review of the academic literature and on interviews with demolition and recycling managers and workers and visits to their sites. 
Assessment of nanomaterial risk is difficult due to the uncertainties over the toxicity of particular nanomaterials and the lack of information on which nanomaterials are contained in which products.

There is some evidence that exposure to nanoparticles from engineered nanomaterials is likely to be low during demolition and construction processes as nanoparticles remain attached to fragments of the underlying matrix. However the evidence base is small and material ageing might also influence this, particularly in the demolition context. Additional exposures may occur as nano-enabled products are recycled and incorporated into new materials.

Measurement of exposure to nanomaterials is complex and data are currently limited. Qualitative assessments methods can be useful as they allow assessments to be made without such measurements, for example categorising activities as low, medium or high risk based on general principles. Such methods tend to reach relatively cautious conclusions, specifying higher levels of protection than expert assessment. Nanomaterial risk may not add substantially to existing construction health hazards (for example silica dust), and commonly used protective methods are likely to be appropriate in many cases.

\section{Building on the findings of this research}

Research objective: To produce guidance for IOSH practitioners and industry stakeholders on nanotechnologies in the built environment

Research findings: Parallels have been drawn between asbestos and some nanomaterials, particularly certain CNTs which have similar needle-like dimensions and bio persistence. In addition, the fact that nanomaterials, like asbestos before, are heralded as being a new 'miracle' solution raises anxieties in some quarters. However, CNTs in particular, and other nano-objects and nanomaterials in general, are very varied in terms of their functionality and their health risk potential. The question 'are nanomaterials hazardous?' is therefore difficult to answer given the wide variability between different substances. The rapid development of materials and new uses for these, and the limited availability of published information from manufacturers further complicates the situation.

It is unlikely that legislative change will have a major impact on this in the short term. Improved clarity will only occur if manufacturers take action to share information more readily and to follow voluntary guidance to provide greater detail in safety data sheets regarding any nano-objects. Action by manufacturers to design products and materials which are intrinsically safer is the best route to minimise any risks to workers in the long term (for example, CNTs which are non-biopersistent and are short or tangled) and research is ongoing to explore this; as well as to better understand the toxicity of nanomaterials and the specific factors which influence this. Further research is needed to assess the potential for worker exposure from nano-objects in construction and demolition and to consider the impact of secondary nanomaterials.

The use of most currently available products which contain nanomaterials is unlikely to add substantially to the risks already present for those working in construction and demolition. In the absence of detailed information, the following steps are recommended:

- robust management of existing health risks, which continue to be significant, notwithstanding any novel risks from nanomaterials;

- the use of a precautionary approach, extending the control measures already taken to protect against known risks to reduce exposures to those where less is known about toxicity; 
- asking questions of suppliers and manufacturers regarding the exact content of new products. This is particularly important where fibres may be present as the size and shape of these can influence risk profiles substantially; and

- recording the materials/products used (for example in the CDM file or through the Building Information Model - BIM) to facilitate decision making during subsequent refurbishment or demolition.

Nanomaterials have much to offer the construction industry, and their use is expected to grow substantially in the future. If good practice and the right controls are in place and used correctly, for all existing hazardous substances, then the introduction of most currently available nanomaterials is unlikely to significantly increase the risk or require additional control measures. Nevertheless, it is important that we balance the benefits and the opportunities for improved functionality with a degree of caution. This includes an ongoing research agenda to improve understanding; challenge to manufacturers and suppliers to improve clarity and transparency; and a questioning approach by the industry. This will enable us to continue making well informed judgements to protect the workforce in the longer term. 